\title{
Quantitative multivolume proton- magnetic resonance imaging in patients with cystic fibrosis lung disease: comparison with clinical indicators
}

\author{
Francesca Pennati ${ }^{1}$, Caterina Salito ${ }^{1}$, Irene Borzani ${ }^{2}$, Giulia Cervellin ${ }^{2}$, \\ Simone Gambazza (10) ${ }^{3,4}$, Riccardo Guarise ${ }^{4}$, Maria Chiara Russo ${ }^{4}$, \\ Carla Colombo ${ }^{4}$ and Andrea Aliverti ${ }^{1}$
}

Affiliations: ${ }^{1}$ Dipartimento di Elettronica, Informazione e Bioingegneria, Politecnico di Milano, Milan, Italy. ${ }^{2}$ Radiologia Pediatrica, Fondazione IRCCS Ca' Granda Ospedale Maggiore Policlinico, Milan, Italy. ${ }^{3}$ U.O.C. Direzione Professioni Sanitarie, Fondazione IRCCS Ca' Granda Ospedale Maggiore Policlinico, Milan, Italy. ${ }^{4}$ Cystic Fibrosis Centre, Fondazione IRCCS Ca' Granda Ospedale Maggiore Policlinico, Milan, Italy. ${ }^{5}$ Dept of Pathophysiology and Transplantation, Università degli Studi di Milano, Milan, Italy.

\section{Correspondence:}

Francesca Pennati, Dipartimento di Elettronica, Informazione e Bioingegneria, Politecnico di Milano, Piazza L. da Vinci, 32, 20133 Milano, Italy.

E-mail: francesca.pennatiđpolimi.it

@ERSpublications

MRI biomarkers of ventilation impairment correlate with spirometry measurements, lung clearance index and lung structure alterations in cystic fibrosis http://bit.ly/2Vbg1DV

Cite this article as: Pennati F, Salito C, Borzani I, et al. Quantitative multivolume proton-magnetic resonance imaging in patients with cystic fibrosis lung disease: comparison with clinical indicators. Eur Respir J 2019; 53: 1702020 [https://doi.org/10.1183/13993003.02020-2017].

ABSTRACT This cross-sectional study aims to verify the relationship between quantitative multivolume proton-magnetic resonance imaging $\left({ }^{1} \mathrm{H}-\mathrm{MRI}\right)$ and clinical indicators of ventilatory abnormalities in cystic fibrosis (CF) lung disease.

Non-enhanced chest MRI, spirometry and multiple breath washout was performed by 28 patients (10-27 years) with CF lung disease. Images acquired at end-inspiration and end-expiration were registered by optical flow to estimate expiratory-inspiratory proton-density change $\left(\Delta^{1} \mathrm{H}-\mathrm{MRI}\right)$ as a measure of regional ventilation. Magnetic resonance images were also evaluated using a CF-specific scoring system.

Biomarkers of $\mathrm{CF}$ ventilation impairment were defined from the $\Delta^{1} \mathrm{H}$-MRI as follows: $\Delta^{1} \mathrm{H}-\mathrm{MRI}$ median, $\Delta^{1} \mathrm{H}$-MRI quartile coefficient of variation (QCV) and percentage of low-ventilation volume (\%LVV). Imaging biomarkers correlated to all the clinical measures of ventilation abnormality, with the strongest correlation between $\Delta^{1} \mathrm{H}-\mathrm{MRI}$ median and forced expiratory volume in $1 \mathrm{~s}\left(\mathrm{r}^{2}=0.44, \mathrm{p}<0.001\right)$, $\Delta^{1} \mathrm{H}-$ MRI QCV and lung clearance index (LCI) $\left(\mathrm{r}^{2}=0.51, \mathrm{p}<0.001\right)$ and \%LVV and LCI $\left(\mathrm{r}^{2}=0.66, \mathrm{p}<0.001\right)$. Correlations were also found between imaging biomarkers of ventilation and morphological scoring.

The study showed a significant correlation between quantitative multivolume MRI and clinical indicators of CF lung disease. MRI, as a non-ionising imaging technique, may be particularly attractive in CF care for longitudinal evaluation, providing a new imaging biomarker to detect early ventilatory abnormalities. 


\section{Introduction}

Lung disease is the most frequent cause of morbidity and mortality in patients with cystic fibrosis (CF) $[1,2]$. With new therapies targeting and improving outcomes in younger CF populations and subgroups of patients with specific CF genotypes, there is an evident need for new sensitive markers of early changes in lung structure and function $[3,4]$. Imaging lung function and structure is particularly important in $\mathrm{CF}$ because functional lung changes are dissociated from structural changes [5, 6]. Moreover, given that $\mathrm{CF}$ changes are not evenly distributed through the lung, with large areas of normal lung tissue adjacent to areas of localised structural changes, imaging may reveal the structure-function relationship at the regional and whole lung level [7].

Functional outcomes of CF lung disease are traditionally measured with spirometry and functional tests. Forced expiratory volume in $1 \mathrm{~s}\left(\mathrm{FEV}_{1}\right)$ is the most widely used endpoint in clinical trials. Recently, forced expiratory flow at $25-75 \%$ of forced vital capacity $(\mathrm{FEF} 25-75 \%)$ and forced expiratory flow at $75 \%$ of forced vital capacity (FEF75\%) have gained new interest as markers of early lung disease, being more sensitive than FEV 1 in detecting obstruction of the small airways $[8,9]$. Nevertheless, spirometry parameters have limited sensitivity for regional abnormalities and provide no information on structural impairment. Moreover, FEV1 is effort dependent, making it unsuitable for the assessment of lung disease in patients aged $<6$ years $[10,11]$. The lung clearance index (LCI), an effort-independent measure of ventilation inhomogeneity derived from the multiple breath washout (MBW) technique, has been advocated as an early sensitive marker of CF lung disease $[12,13]$. LCI is an earlier indicator of disease progression than spirometry [14] and correlates with structural damage [15, 16]. LCI can detect treatment responses to interventions $[17,18]$, and has been used as a primary outcome in the context of a randomised controlled trial [19].

High-resolution computed tomography (HRCT) is currently the most sensitive method of detecting and monitoring structural lung changes in CF [20,21]. However, the use of computed tomography (CT) in paediatric patients and for lifelong follow-up is restricted by the cumulative radiation dose [22]. Recently, magnetic resonance imaging (MRI) has been established as a radiation-free alternative to CT to detect early structural abnormalities of the CF lung [23, 24]. HRCT and MRI scoring systems have yielded sensitive markers to quantify and characterise the structural changes at various stages of the disease [25-27]. Nevertheless, scoring systems are not routinely applied in most clinics because they are time-consuming, subjective and require training. To date, no automated, validated image analysis system is available to quantify the structural impairment in CF lung disease. Systems have been developed with chest CT scans to quantify trapped air $[28,29]$ and airway/artery ratios [30] in CF studies. There is a need to develop automated and more sensitive image analysis methods on chest MRI.

Recently, non-enhanced proton-MRI ( $\left.{ }^{1} \mathrm{H}-\mathrm{MRI}\right)$ has been proposed to quantify regional ventilation by measuring differences in proton signal intensity between breath-hold images acquired in inspiration and expiration [31]. The technique has been developed for use in a standard clinical setting, based on gradient echo acquisitions on a $1.5 \mathrm{~T}$ magnetic resonance (MR) unit [32]. The Fourier decomposition (FD) technique is an alternative method to image ventilation; this spectrally separates the signal originating from the pulmonary and the cardiac cycle in a series of ${ }^{1} \mathrm{H}$ MR images acquired in free-breathing [33]. Multiple advances in this technique have improved the stability of the original FD method, e.g. the matrix pencil decomposition technique [34] and self-gated non-contrast-enhanced functional lung imaging (SENCEFUL) [35].

We assumed that breath-hold multivolume ${ }^{1} \mathrm{H}-\mathrm{MRI}$ would provide quantitative biomarkers in CF lung disease capable of detecting functional impairment. In particular, we aimed to verify the correlation between multivolume ${ }^{1} \mathrm{H}$-MRI biomarkers and standard clinical parameters, and to investigate the relationship between functional impairment and structural damage, quantified by a specific CF-scoring system.

\section{Materials and methods}

Study subjects

In this cross-sectional study, patients with CF who were admitted for their annual review in a stable clinical condition were consecutively recruited in the Lombardia Region CF Centre, University of Milan, Fondazione IRCCS Ca' Granda Ospedale Maggiore Policlinico, Milan, Italy. Diagnosis of CF was confirmed by a sweat chloride level $>60 \mathrm{mM}$ and/or two CF-causing mutations in the CFTR gene according to established diagnostic criteria. All patients underwent chest MRI, spirometry and a MBW test as part of their annual surveillance programme.

The study was approved by the local ethics committee and conducted in compliance with the guidelines of the institutional review board; written informed consent was obtained from each patient or a legal guardian. 


\section{Spirometry}

Spirometry was performed on the same day as MRI and included the measurement of FEV1, forced vital capacity (FVC), FEF75\% and $\mathrm{FEF} 25-75 \%$. Values were expressed as a percentage of predicted values according to the prediction equations of the Global Lung Function Initiative [36]. Spirometry was performed in compliance with American Thoracic Society/European Respiratory Society (ATS/ERS) guidelines [37].

\section{Multiple breath washout}

MBW was performed within 15 days of MRI. The commercial nitrogen-MBW equipment ExhalyzerD and associated software package (Eco-Medics AG, Duernten, Switzerland) were used for data acquisition, storage and analysis in compliance with the standard operating procedure [38]. LCI was defined in compliance with the ATS/ERS consensus statement [39] as the number of lung volume turnovers (i.e. the cumulative expired volume divided by the functional residual capacity) required to reduce the $\mathrm{N}_{2}$ end-tidal concentration to $1 / 40$ th of the starting concentration.

\section{${ }^{1} H-M R I$ acquisition}

Subjects were imaged with a $1.5 \mathrm{~T}$ MR scanner (Magnetom AVANTO; Siemens, Erlangen, Germany) at suspended full end-expiration (EXP), approximating residual volume (RV), and suspended full end-inspiration (INSP), approximating total lung capacity (TLC). The standard MR protocol used routinely at our institution for the follow-up of lung involvement in CF patients includes the following sequences: 1) T2-weighted half-Fourier single-shot turbo spin echo (HASTE); 2) T2-weighted turbo spin echo using the periodically rotated overlapping parallel lines with enhanced reconstruction technique (TSE-PROPELLER), with end-expiratory triggered acquisition; 3) T2-weighted balanced steady-state free precession (bSSFP), acquired in free-breathing; and 4) T1-weighted three-dimensional gradient echo (3D GRE), with breath-hold of about $6 \mathrm{~s}$. Sequence parameters are reported in table 1. All patients were experienced in performing respiratory manoeuvres because they frequently underwent spirometric tests during their follow-up. Before the imaging session they were instructed to sustain TLC and RV volumes during the scan. Images were checked by the radiologist and re-acquired within the same imaging session if they were not satisfactory.

\section{Image processing}

From the top diaphragm to the aortic arch, six corresponding levels were selected in the expiratory and inspiratory T1-weighted 3D GRE scans. The images to be registered were accurately selected at the same apical-caudal level, i.e. at the same level of the airways' and vessels' trees, to minimise through-plane motion. To obtain maps of ${ }^{1} \mathrm{H}$-density difference between the two lung volumes, the original MRI scans were processed as follows:

1) Semi-automatic lung segmentation was performed to separate lung parenchyma from the surrounding soft tissues, using the software MIPAV (Medical Image Processing, Analysis and Visualization) [40]. Image selection and semi-automatic segmentation required about $10 \mathrm{~min}$ per patient.

2) Images were normalised to the mean thoracic soft-tissue signal to quantify the relative lung signal.

3) To follow ${ }^{1} \mathrm{H}$ signal changes between the expiratory and inspiratory images, deformable image registration (DIR), based on the optical flow algorithm [41], was applied to map corresponding pixels in the expiratory and the inspiratory image. To make the registration process more sensitive to

TABLE 1 Proton-magnetic resonance imaging parameters for the acquisition sequences

\begin{tabular}{|c|c|c|c|c|c|c|c|}
\hline Sequence & TR ms & TE ms & $\begin{array}{c}\text { Slice } \\
\text { thickness } \mathrm{mm}\end{array}$ & $\begin{array}{l}\text { In-plane resolution } \\
\qquad m \times m \times m\end{array}$ & $\begin{array}{l}\text { Flip } \\
\text { angle }\end{array}$ & Respiratory phase & TA $s$ \\
\hline T2-HASTE & 900 & 85 & 6 & $1.4 \times 1.4$ & 171 & INSP & 18 \\
\hline T2-bSSFP & 3.61 & 1.46 & 4 & $1.1 \times 1.1$ & 52 & Free-breathing & 22 \\
\hline T1-3D GRE & 3.18 & 1.05 & 5 & $0.9 \times 0.9$ & 5 & EXP, INSP & 6 \\
\hline
\end{tabular}

TR: repetition time; TE: echo time; TA: total acquisition time; T2-HASTE: T2-weighted half-Fourier single-shot turbo spin echo; T2-TSE-PROPELLER: T2-weighted turbo spin echo using the periodically rotated overlapping parallel lines with enhanced reconstruction technique; T2-bSSFP: T2-weighted balanced steady-state free expiration; T1-3D GRE: T1-weighted three-dimensional gradient echo; INSP: inspiration; EXP: expiration. 
structures rather than to overall intensity, which changes with lung volume, a Laplacian filter was applied to the images prior to DIR [32].

4) The registered images were subtracted pixel by pixel to provide a map of local ${ }^{1} \mathrm{H}$ signal change between the two lung volumes $\left(\Delta^{1} \mathrm{H}-\mathrm{MRI}={ }^{1} \mathrm{H}_{\mathrm{EXP}}-{ }^{1} \mathrm{H}_{\mathrm{INSP}}\right)$.

Values are expressed as a percentage of the mean thoracic soft-tissue MRI signal.

\section{MR image analysis \\ MRI measures of regional ventilation}

For each patient, the following MRI functional parameters were calculated across the six lung levels: 1) $\Delta^{1} \mathrm{H}$-MRI median; 2) $\Delta^{1} \mathrm{H}$-MRI quartile coefficient of variation (QCV), as an index of spatial heterogeneity; and 3) percentage of low-ventilated volume (\%LVV), as an index of total ventilation defect.

We defined \%LVV as the percentage of pixels characterised by $\Delta^{1} \mathrm{H}-\mathrm{MRI}<5$ [42] and 1) a low signal magnitude in the expiratory and inspiratory images, i.e. ${ }^{1} \mathrm{H}-\mathrm{MRI}_{\mathrm{EXP}}<10 \%$ and ${ }^{1} \mathrm{H}-\mathrm{MRI}_{\mathrm{INSP}}<10 \%$, or 2 ) a high signal magnitude in the expiratory and inspiratory images, i.e. ${ }^{1} \mathrm{H}-\mathrm{MRI}_{\mathrm{EXP}}>50 \%$ and ${ }^{1} \mathrm{H}-\mathrm{MRI}_{\mathrm{INSP}}>50 \%$. The threshold for low signal magnitude (10\% of the mean thoracic soft-tissue signal) corresponds to the mean signal of the trachea across all patients.

\section{MRI morphological scoring}

The combination of T2- and T1-weighted sequences allows for tissue characterisation. MR images were morphologically scored by a single radiologist (IB, with 8 years of experience in pulmonary MRI) at two time points (at a 12-week interval) in compliance with previously published scoring systems [26, 27]. The score for perfusion was excluded because contrast medium is not routinely used at our institution. All identifying information was removed and the images were read in random order. The lungs were divided into six lobar regions (five lobes plus lingula) and assessed for 1) bronchiectasis/bronchial wall thickening (bronchial wall abnormalities); 2) mucus plugging; 3) abscesses/sacculations; 4) consolidations; and 5) special findings. Findings were assessed for each individual lobe as 0 (no lobe involvement), 1 (less than half a lobe involved) and 2 (more than half a lobe involved). Intra-reader agreement for total morphological score was assessed.

Figure 1 illustrates the steps of the overall image processing and analysis algorithm, as follows: 1) original MRI scans acquired at RV and TLC; 2) segmentation of lung parenchyma; 3) normalisation of the lung parenchyma; 4) application of DIR to deform the RV onto the TLC image; 5) pixel-by-pixel subtraction of the TLC image from the registered RV image to map local ${ }^{1} \mathrm{H}$ signal change and computation of $\Delta^{1} \mathrm{H}-\mathrm{MRI}$ median and QCV; and 6) computation of the LVV, expressed as a percentage of the total volume.

All algorithms for image processing and quantitative analysis were implemented using bespoke software developed in MATLAB (The MathWorks, Inc., Natick, MA, USA).

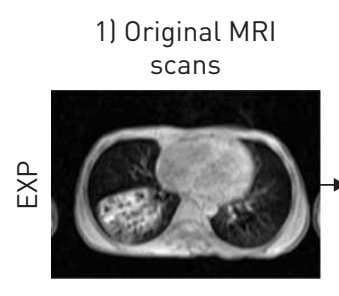

2) Segmentation

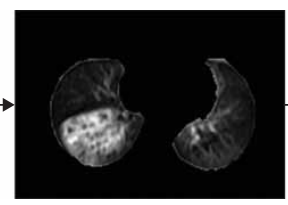

3) Normalisation
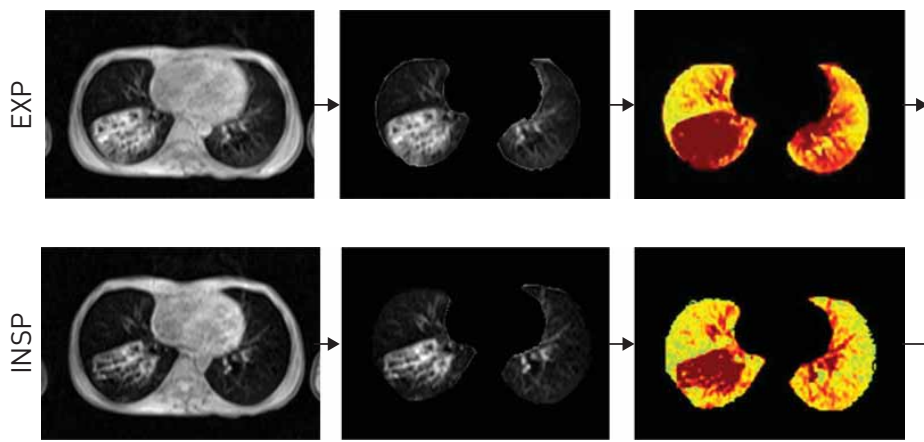

4) Image registration

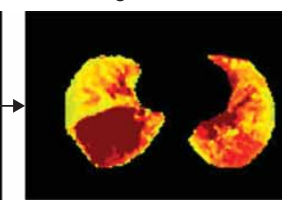

5) $\Delta^{1} H-M R I$

6) \%LVV

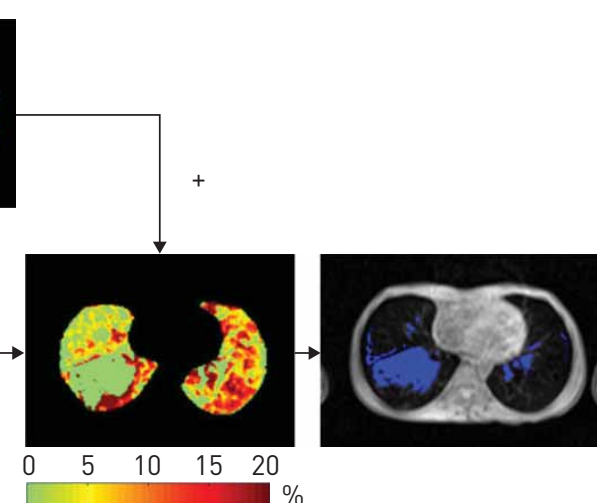

FIGURE 1 Image processing steps. 1) The original magnetic resonance imaging (MRI) scans acquired at suspended full end-expiration (EXP) and suspended full end-inspiration (INSP) selected at the same lung level; 2) image segmentation to extract the lungs from the surrounding tissues; 3) image normalisation to mean thoracic soft-tissue signal; 4) application of deformable image registration to deform the EXP onto the INSP image; 5) pixel-by-pixel subtraction of the registered INSP image from the EXP image, to provide a map of local ${ }^{1} \mathrm{H}$ signal change between the two lung volumes $\left.\left(\Delta^{1} \mathrm{H}-\mathrm{MRI}={ }^{1} \mathrm{H}_{\mathrm{EXP}}-{ }^{1} \mathrm{H}_{\text {INSP }}\right) ; 6\right)$ calculation of percentage low-ventilation volume (\%LVV), as a percentage of total lung volume; low-ventilated pixels are superimposed onto the original INSP image as blue pixels. 


\begin{tabular}{|c|c|c|c|c|c|c|c|c|c|}
\hline Subject & Age years & Sex ${ }^{\#}$ & BMI & CF genotype & Sputum culture & FEV $1 \%$ pred & FVC $\%$ pred & FEF $25-75 \% \%$ pred & LCl \\
\hline 1 & 16 & $\mathrm{~F}$ & 17.7 & F508del, UN & Bcc, A.fum & 49.7 & 71.9 & 15.1 & 16.6 \\
\hline 2 & 12 & $\mathrm{~F}$ & 15 & F508del, F508del & $\mathrm{Ax}, \mathrm{Hi}$ & 54.1 & 62.8 & 27.3 & 23.7 \\
\hline 3 & 14 & $\mathrm{~F}$ & 17.7 & $3849+10 \mathrm{KbC}>\mathrm{T}, 711+5 \mathrm{G}->\mathrm{A}$ & $\mathrm{Pa}$ & 47.6 & 60.7 & 27.3 & 13.0 \\
\hline 4 & 19 & $\mathrm{~F}$ & 25.1 & F508del, F508del & $\mathrm{Pa}, \mathrm{MSSA}$ & 112.7 & 126.7 & 73.1 & 11.6 \\
\hline 5 & 17 & M & 19.5 & F508del, UN & MSSA, $\mathrm{Pa}$ & 103.4 & 100.9 & 99.5 & 14.3 \\
\hline 6 & 15 & $\mathrm{~F}$ & 19 & F508del, N1303K & $\mathrm{Pa}$ & 131.2 & 129.5 & 113.9 & 11.1 \\
\hline $7^{\text {ก }}$ & 17 & M & 21.5 & G542X, G542X & MSSA, Sm, A.ver & 107.6 & 112.1 & 85.3 & 12.7 \\
\hline $8^{\pi}$ & 17 & M & 21.5 & G542X, G542X & MSSA, Sm, A.ver & 107.6 & 112.1 & 85.3 & 12.7 \\
\hline 9 & 16 & M & 16.5 & F508del, N1303K & MSSA, Pa, Mabsc & 76.9 & 100.2 & 35.0 & 16.7 \\
\hline 10 & 17 & M & 17.6 & 5T-TG12, L61P & N.spp & 48.6 & 82.9 & 17.8 & 13.2 \\
\hline 11 & 17 & $\mathrm{~F}$ & 21.4 & F508del, F508del & S.spp & 74.1 & 88.1 & 38.8 & 17.2 \\
\hline 12 & 11 & M & 14.8 & F508del, R1066C & $\mathrm{Pa}, \mathrm{PN}$ & 100.6 & 106.0 & 73.9 & 10.1 \\
\hline 13 & 17 & $\mathrm{~F}$ & 22.2 & F508del, 3659delC & A.fum, MSSA, Pa & 78.3 & 80.6 & 64.4 & 14.6 \\
\hline 14 & 14 & M & 16.7 & F508del, F508del & $\mathrm{Pa}, \mathrm{Mabsc}$ & 106.6 & 111.0 & 85.4 & 13.1 \\
\hline 15 & 20 & $\mathrm{~F}$ & 18.2 & F508del, F508del & Ax, MSSA & 82.1 & 88.5 & 60.5 & 12.9 \\
\hline 16 & 13 & M & 15.2 & F508del, G85E & $\mathrm{Pa}, \mathrm{MSSA}$ & 69.9 & 79.9 & 41.4 & 13.2 \\
\hline 17 & 13 & M & 14.3 & F508del, G85E & $\mathrm{Pa}, \mathrm{MSSA}$ & 64.2 & 76.7 & 32.5 & 14.7 \\
\hline 18 & 25 & M & 22.9 & N1303K, 2183AA $>$ G & A.fum, Pa, A.flav & 59.6 & 82.2 & 20.4 & 20.1 \\
\hline 19 & 15 & $\mathrm{~F}$ & 18.2 & F508del, N1303K & MSSA, Sm, A.flav & 55.9 & 93.6 & 17.2 & 21.7 \\
\hline 20 & 23 & M & 22.6 & F508del, F508del & Ax, MSSA & 36.0 & 62.2 & 10.7 & 20.0 \\
\hline 21 & 25 & M & 22.3 & F508del, F508del & $\mathrm{Pa}$ & 84.2 & 97.0 & 49.3 & 11.5 \\
\hline 22 & 24 & $\mathrm{~F}$ & 23.7 & F508 del, N1303K & MSSA, A.fum, A.flav & 102.6 & 108.8 & 79.9 & 11.3 \\
\hline 23 & 26 & $\mathrm{~F}$ & 21.1 & F508Del, F508del & C.spp, A.fum & 55.6 & 82.0 & 18.9 & 20.8 \\
\hline 24 & 10 & $\mathrm{~F}$ & 14.8 & F508del, 1717-1G->A & S.marcescens & 75.5 & 95.6 & 32.2 & 13.2 \\
\hline 25 & 22 & $\mathrm{~F}$ & 21 & F508del, N1303K & MSSA, A.fum & 79.2 & 95.9 & 37.7 & 14.8 \\
\hline 26 & 27 & M & 22.6 & R347P, R1066C & Ax, MSSA & 60.1 & 93.0 & 24.3 & 16.5 \\
\hline 27 & 13 & $\mathrm{~F}$ & 15.6 & G85E, H1375P & MSSA & 103.8 & 98.4 & 119.7 & 8.9 \\
\hline 28 & 16 & M & 17.9 & F508del, G542X & MSSA, Ax, Mabsc & 64.6 & 70.9 & 40.9 & 16.9 \\
\hline Mean $\pm s D$ & $18 \pm 5$ & & $19.2 \pm 3.1$ & & & $78.3 \pm 24.4$ & $91.8 \pm 18.1$ & $51.0 \pm 31.7$ & $14.9 \pm 3.7$ \\
\hline
\end{tabular}

BMI: body mass index; CF: cystic fibrosis; FEV1: forced expiratory volume in $1 \mathrm{~s}$; \% pred: \% predicted; FVC: forced vital capacity; FEF25-75\%: forced expiratory flow at 25-75\% of FVC; LCl: lung clearance index; Bcc: Burkholderia cepacia complex; A.fum: Aspergillus fumigatus group; Ax: Achromobacter xylosoxidans; Hi: Haemophilus influenzae; Pa: Pseudomonas aeruginosa; MSSA: Staphylococcus aureus methicillin sensitive; Sm: Stenotrophomonas maltophilia; A.ver: Aspergillus versicolor; Mabsc: Mycobacterium abscessus complex; N.spp: Nocardia spp.; S.spp: Scedosporium spp.; PN: Streptococcus pneumonia; A.flav: Aspergillus flavus; C.spp: Candida spp.; S.marcescens: Serratia marcescens. ${ }^{\#}$ : $50 \%$ male; ${ }^{\text {: }}$ : patient 7 is the same as patient 8 , with magnetic resonance imaging acquired twice and spirometry and multiple breath washout once within a month. 


\section{Statistical analysis}

Statistical analysis was performed using SigmaStat version 11.0 (Systat Software, San Jose, CA, USA). Associations between MRI functional parameters ( $\Delta^{1} \mathrm{H}-\mathrm{MRI}$ median, QCV and \%LVV) and FEV $1, \mathrm{FVC}$, FEF25-75\% and LCI were assessed by univariate linear regression, with determination of linear correlation coefficients $\left(\mathrm{r}^{2}\right)$. Stepwise forwards regression was performed with MRI functional parameters as the dependent variables, and FEV1, FVC, FEF25-75\% and LCI as independent variables.

Intra-reader agreement for total morphological score was assessed using Bland-Altman analysis. Univariate correlations between MRI morphological scores and FEV1, FVC, FEF25-75\% and LCI were performed using Spearman's rank correlation. In order to investigate any structure-function relationship in CF lung disease, associations between $\Delta^{1} \mathrm{H}-\mathrm{MRI}$ median, QCV and \%LVV and MRI score were assessed by univariate linear regression with determination of the linear correlation coefficients $\left(\mathrm{r}^{2}\right)$.

A p-value $<0.05$ was considered statistically significant.

\section{Results}

In total, 28 consecutively selected patients (mean age 18 years, range 10-27 years, 14 males) were enrolled in the study. All subjects successfully completed the MRI protocol. Patient characteristics, spirometry and MBW are reported in table 2. In one patient, MRI was acquired twice and spirometry and MBW once within a month; this is reported in the table as patient \#7 and \#8 (the second MRI was performed after 2 weeks of intravenous antibiotic therapy owing to an exacerbation).

\section{Comparison between MRI markers of ventilation impairment, spirometry and MBW}

In the overall population, $\Delta^{1} \mathrm{H}$-MRI median ranged from $3.9 \%$ to $17.6 \%$ and $\Delta^{1} \mathrm{H}$-MRI QCV from $0.4 \%$ to $1.4 \%$. The total extent of \%LVV varied from $3 \%$ to $19 \%$.

Regression analysis showed that MRI markers of ventilation impairment related to spirometric measures and LCI (figure 2). $\Delta^{1} \mathrm{H}$-MRI median correlated positively with spirometric measures and negatively with LCI,

a)
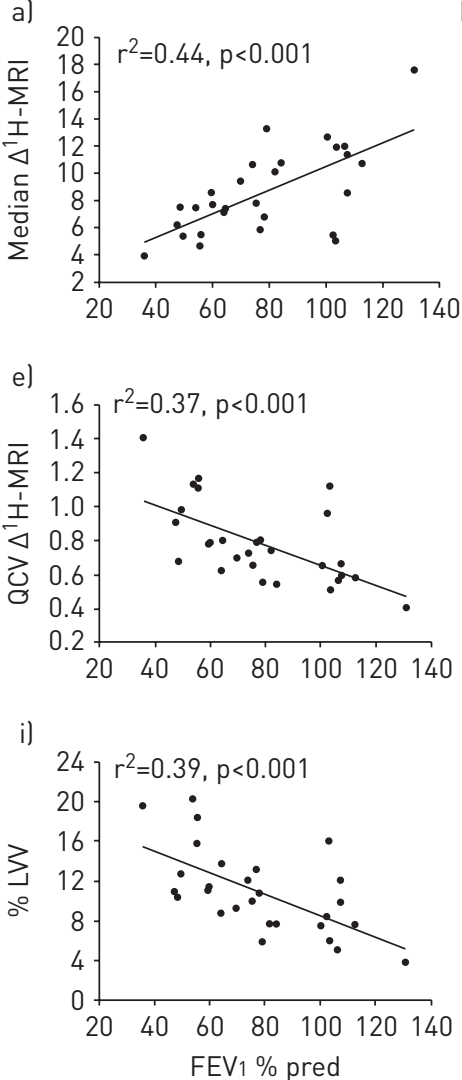

b)
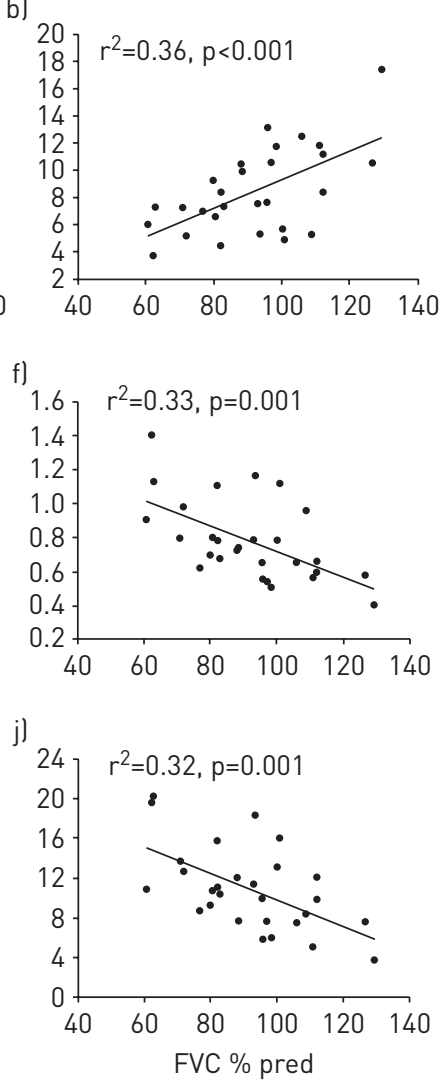

c)
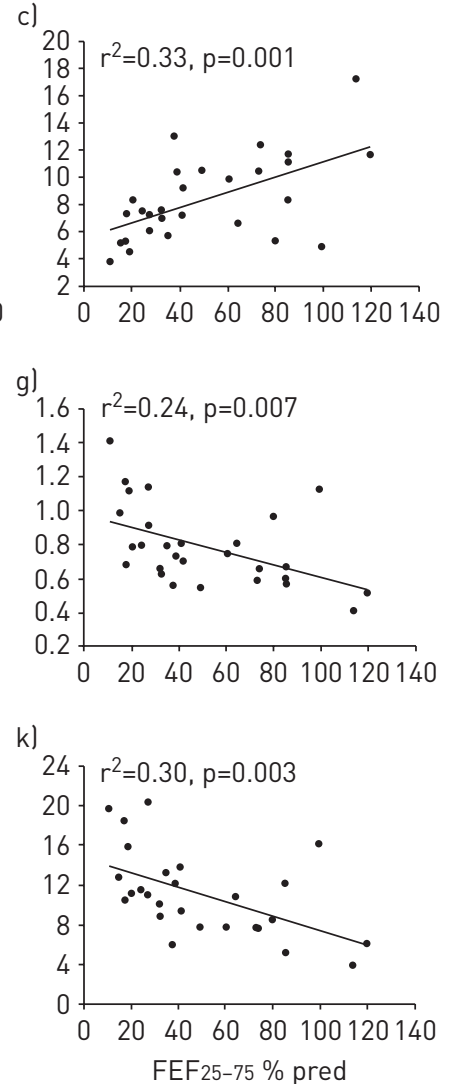

d)
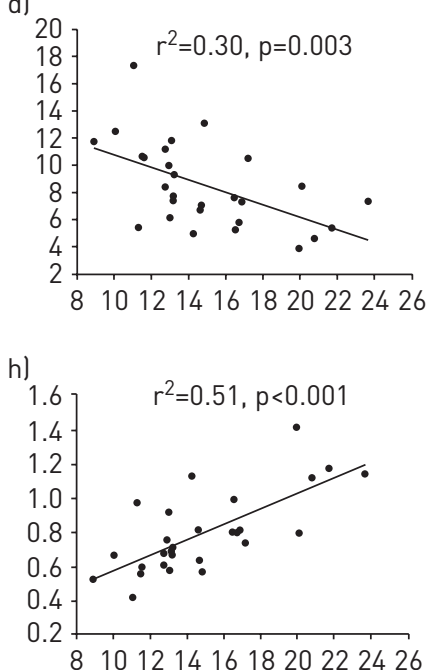

()

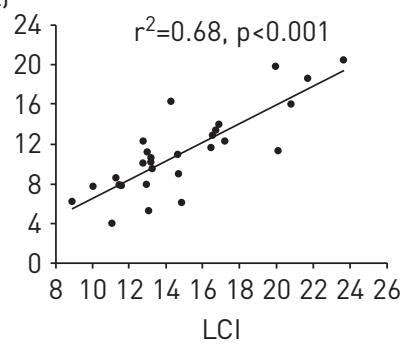

FIGURE 2 Results of linear regression between magnetic resonance imaging (MRI) measures of ventilation and forced expiratory volume in $1 \mathrm{~s}$ (FEV 1 ) \% predicted, forced vital capacity (FVC) \% pred, forced expiratory flow at 25-75\% of FVC (FEF25-75\%) \% pred and lung clearance index (LCI)

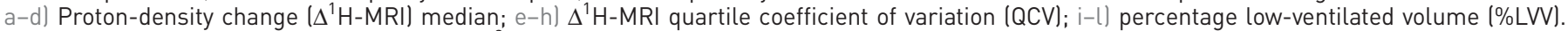
The quadratic linear correlation coefficients $\left(r^{2}\right)$ and $p$-values are reported for each graph. 
with the stronger relationship with FEV1 $\left(\mathrm{r}^{2}=0.44, \mathrm{p}<0.001\right) . \Delta^{1} \mathrm{H}-\mathrm{MRI}$ QCV correlated negatively with spirometric measures and positively with LCI, with the stronger relationship with LCI $\left(\mathrm{r}^{2}=0.51, \mathrm{p}<0.001\right)$. \%LVV correlated negatively with spirometric measures and positively with LCI, with the stronger relationship with LCI $\left(\mathrm{r}^{2}=0.66, \mathrm{p}<0.001\right)$.

Results of the stepwise forwards regression analysis showed FEV1 to be the only independent predictor of $\Delta^{1} \mathrm{H}$-MRI median and LCI to be the only independent predictor of $\Delta^{1} \mathrm{H}-\mathrm{MRI}$ QCV and \%LVV. Figure 3 illustrates the $\Delta^{1} \mathrm{H}$-MRI maps of four representative patients at six equally spaced lung levels from the aortic arch (I-AA) to the top diaphragm (VI-TD). Spirometric measures decrease and LCI increases from panel a to $\mathrm{d}$. Likewise, $\Delta^{1} \mathrm{H}$-MRI decreases from panel a to $\mathrm{d}$, with values up to $20 \%$ homogeneously distributed at all lung levels in patient \#6 (figure 3a) and below 5\% with a lot of heterogeneity in patient \#20 (figure 3d).

Figure 4 shows the \%LVV maps of the four patients reported in figure 3. Low-ventilated regions are coloured blue and superimposed on the original inspiratory MR scans. \%LVV increased from panel a to d, at $3 \%$ in patient $\# 6,7 \%$ in patient $\# 21,13 \%$ in patient $\# 28$ and $18 \%$ in patient $\# 20$.

Total morphological score showed good intra-reader agreement (mean difference 0.07 , limits of agreement 2.7-2.5). The individual morphological MRI scores in the overall population are reported in table 3. Total score ranged from 4 to 25 , with a higher occurrence and severity of bronchial wall thickening/ bronchiectasis and mucus plugging (respectively present in 28 and 27 patients with subscores up to 11 and 9). Correlation analysis showed that the total morphological score correlated with FEV1, FVC, FEF25-75\% and LCI $\left(\mathrm{r}^{2}=0.28,0.29,0.24\right.$ and $\left.0.47 ; \mathrm{p}<0.01\right)$, as well as the subscores of bronchial wall thickening/ bronchiectasis, mucus plugging and consolidation.

Relationship between MRI score and MRI markers of ventilation

Figure 5 reports the linear regression analysis between $\Delta^{1} \mathrm{H}-\mathrm{MRI}$ median (figure $5 \mathrm{a}$ ), QCV (figure $5 \mathrm{~b}$ ) and \%LVV (figure 5c) across the six lung levels and total MRI score. With increasing MRI score, $\Delta^{1} \mathrm{H}-\mathrm{MRI}$ median decreased $\left(\mathrm{r}^{2}=0.23, \mathrm{p}=0.01\right), \Delta^{1} \mathrm{H}-\mathrm{MRI}$ QCV increased $\left(\mathrm{r}^{2}=0.42, \mathrm{p}<0.001\right)$ and \%LVV increased $\left(\mathrm{r}^{2}=0.47, \mathrm{p}<0.001\right)$.

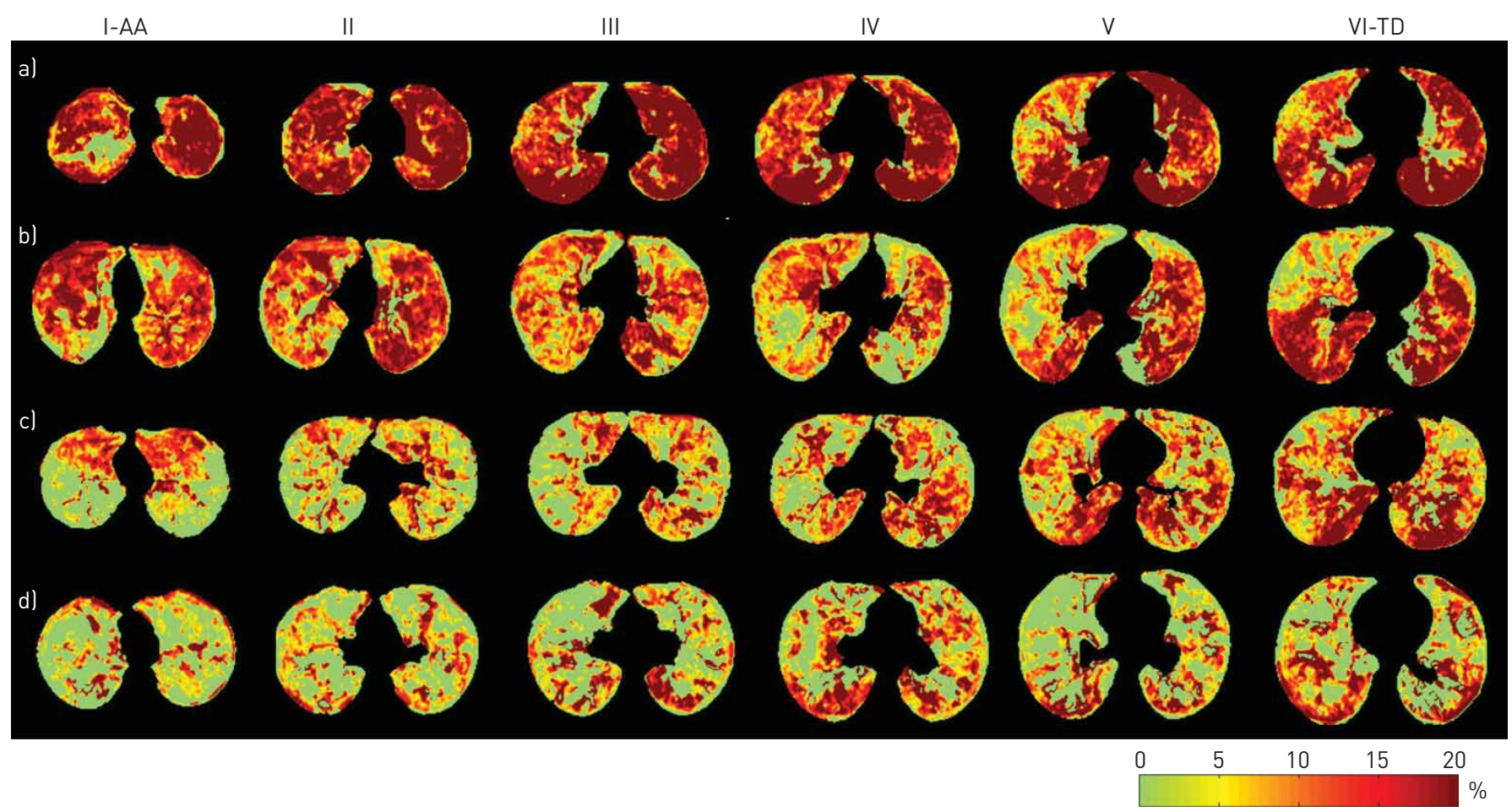

FIGURE 3 Proton-density change $\left(\Delta^{1} \mathrm{H}-\mathrm{MRI}\right)$ maps of four representative patients (a-d) are shown at six equally spaced lung levels from the aortic arch (I-AA) to the top diaphragm (VI-TD). From a to $d$ the patients have decreased forced expiratory volume in $1 \mathrm{~s}$ (FEV 1 ) \% predicted and forced vital capacity (FVC) \% pred, and increased lung clearance index (LCI). a) Patient \#6, FEV $1=131 \%, F V C=129 \%, L C l=11 ;$ b) patient \#21, FEV1=84\%, $\mathrm{FVC}=97 \%, \mathrm{LCl}=11$; c) patient \#28, $\mathrm{FEV} 1=65 \%, \mathrm{FVC}=71 \%, \mathrm{LCl}=17$; d) patient $\# 20, \mathrm{FEV} 1=36 \%, \mathrm{FVC}=62 \%, \mathrm{LCl}=20$. Colour spectra indicate the ${ }^{1} \mathrm{H}$ signal difference as a percentage of the mean thoracic soft-tissue signal. 


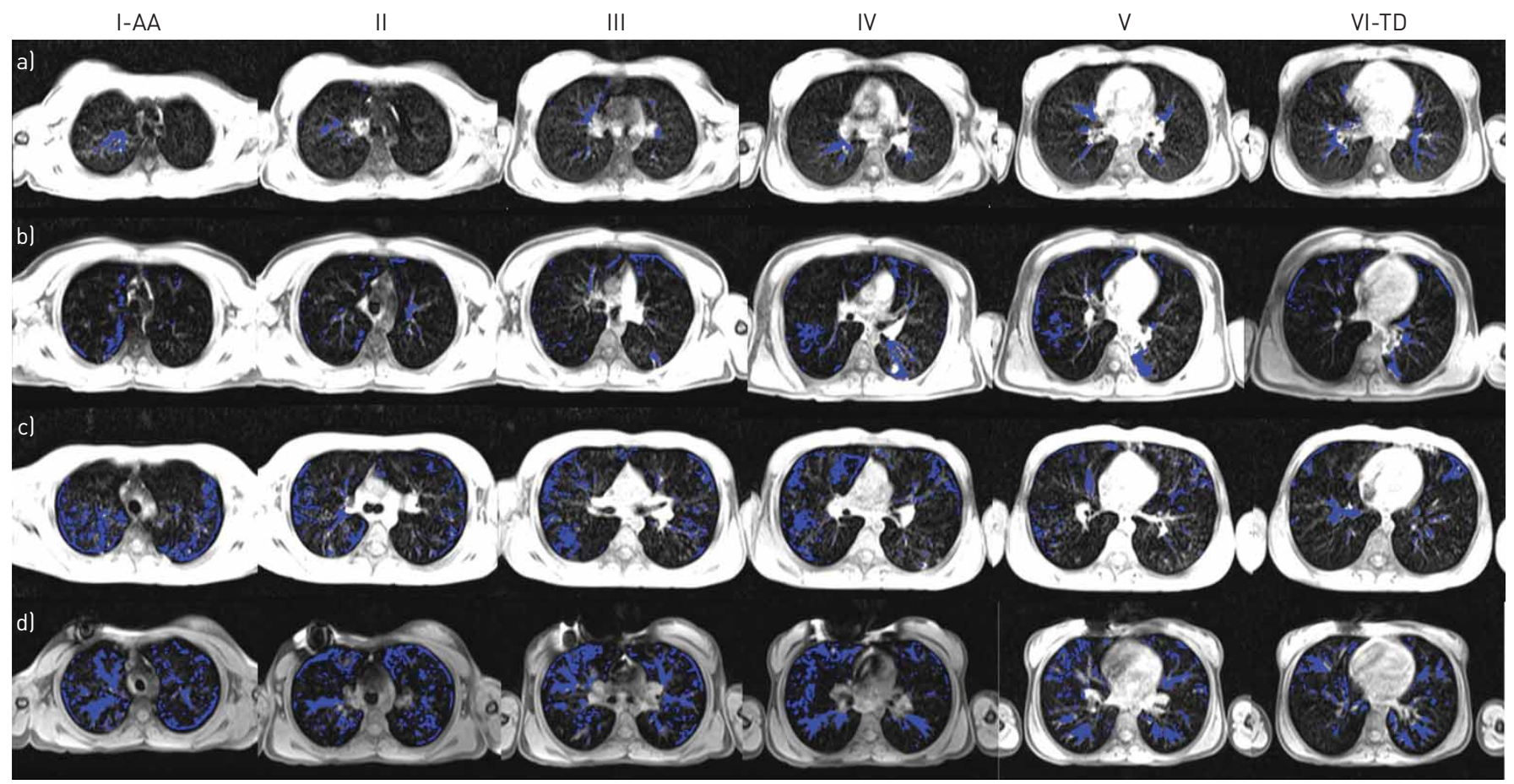

FIGURE 4 The low-ventilated regions of the four representative patients (a-d) shown in figure 3 are coloured in blue and superimposed on the original inspiratory magnetic resonance scans at six equally spaced lung levels from the aortic arch (I-AA) to the top diaphragm (VI-TD). From a to $d$ the percentage low-ventilated volume increases: al patient \#6,3\%; b) patient \#21,7\%; c) patient \#28, 13\%; d) patient \#20, 18\%.

\begin{abstract}
Discussion
This preliminary cross-sectional study evaluates the relationship between non-enhanced multivolume MRI and clinical indicators of disease severity in CF lung disease. Results show that quantitative multivolume MRI strongly correlates with spirometry and MBW technique. MRI has the advantage over global measures of functional impairment that it can identify local structural and functional alterations in CF lung disease. As a non-ionising imaging technique, quantitative multivolume MRI is particularly attractive for longitudinal evaluation in CF care.

First, $\Delta^{1} \mathrm{H}$-MRI correlates to spirometry and to MBW. In CF, the progressive deterioration of lung function and the permanent structural changes result in regions with low/no gas volume change, and thus low/no proton-density change. If total gas volume change decreases, $\Delta^{1} \mathrm{H}$-MRI median decreases. If total gas volume change is more heterogeneously distributed, i.e. there is an increased number of localised functional impairments adjacent to healthy lung areas, $\Delta^{1} \mathrm{H}-\mathrm{MRI}$ QCV increases. This relationship is confirmed by the stronger correlation between $\Delta^{1} \mathrm{H}$-MRI median and spirometric measures, and between $\Delta^{1} \mathrm{H}-\mathrm{MRI} \mathrm{QCV}$ and LCI.
\end{abstract}

TABLE 3 Prevalence of magnetic resonance imaging morphological subscores [30, 31] and univariate correlations between each morphological subscore and outcome

Prevalence $\mathrm{n}(\%) \quad$ Score min-max

Correlation $r^{2}$

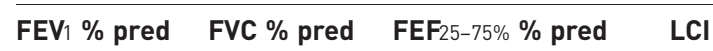

\begin{tabular}{lrrrr}
\hline Bronchial wall thickening/bronchiectasis & $28(100)$ & $1-11$ & $0.17^{*}$ & $0.53^{* * *}$ \\
Mucus plugging & $27(96.4)$ & $0-9$ & $0.15^{*}$ & $0.43^{* * *}$ \\
Abscesses/sacculations & $8(28.6)$ & $0-3$ & & $0.20^{*}$ \\
Consolidation & $19(67.9)$ & $0-4$ & & $0.20^{*}$ \\
Special findings & $15(53.6)$ & $0-5$ & $0.28^{* * *}$ & $0.29^{* * *}$ \\
Total score & $28(100)$ & $4-5$ & $0.24^{* *}$ & $0.47^{* * *}$ \\
\hline
\end{tabular}

FEV1: forced expiratory volume in $1 \mathrm{~s} ; \%$ pred: \% predicted; FVC: forced vital capacity; FEF25-75: forced expiratory flow at 25-75\% of FVC; LCI: lung clearance index. ${ }^{*}: p<0.05 ;{ }^{* *}: p<0.01 ;{ }^{* * *}: p<0.001$. 

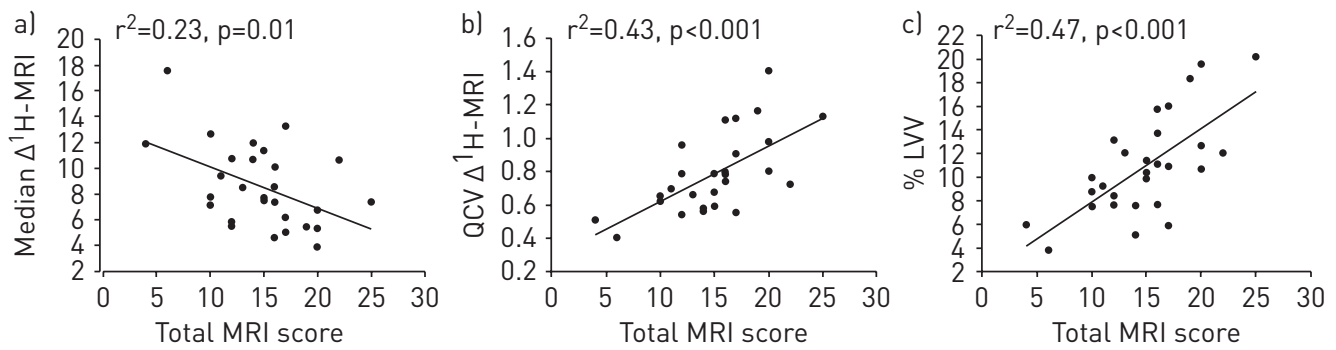

FIGURE 5 Correlation results between magnetic resonance imaging (MRI) measures of ventilation and MRI morphological score. a) Proton-density change ( $\left.\Delta^{1} \mathrm{H}-\mathrm{MRI}\right)$ median; b) $\Delta^{1} \mathrm{H}-\mathrm{MRI}$ quartile coefficient of variation $(\mathrm{QCV})$; c) percentage low-ventilated volume (\%LVV). The quadratic linear correlation coefficients $\left(r^{2}\right)$ and p-values are reported for each graph.

Second, the \%LVV strongly correlates with LCI and moderately correlates with spirometric measures. These results are consistent with the observation that low-ventilated lung units delay the efficiency of gas mixing (thus increasing LCI), contributing to a considerable amount of the expiratory flow limitation (thus decreasing FEV1) [43]. Moreover, the stronger correlation of \%LVV with LCI, rather than with spirometric measures, supports the hypothesis that parallel heterogeneities of specific ventilation preferentially lead to LCI increase [44].

Maps of \%LVV are compatible with the very patchy ventilation images obtained by hyperpolarised gas (either ${ }^{3} \mathrm{He}$ or ${ }^{129} \mathrm{Xe}$ ) MRI in adult and paediatric CF patients [45-47], but the extent of ventilation defects was lower in the present study. We attribute this difference to the higher lung volume variation between the expiratory and inspiratory scans, which was necessary to better differentiate health from disease [32]. In comparison to hyperpolarised gas MRI, multivolume MRI is based on straightforward pulse sequences and hardware. It does not require any gaseous tracer and can be performed on nearly any MRI scanner because images are acquired with a standard "gradient echo" sequence. The current image analysis is performed in two dimensions and requires user intervention for image selection and segmentation. In fact, the structural heterogeneity of the CF lung prevents the use of the $3 \mathrm{D}$ segmentation and registration algorithm [32]. Further software development, with the automation of the segmentation algorithm, would allow automatic 3D image analysis that would be ideal for everyday clinical use. The fact that multivolume MRI can identify local structural and functional alterations may provide early detection ahead of LCI or spirometry and more sensitive monitoring of disease progression.

Recently, non-contrast-enhanced MRI has been investigated in CF lung disease. Expiratory-inspiratory breath-hold MRI and the FD-based SENCEFUL technique have reported significant differences in ventilation between patients with $\mathrm{CF}$ and healthy subjects $[42,48]$ and among CF-specific structural impairments [42]. The FD-based matrix pencil decomposition method has demonstrated associations between ventilation impairment, LCI and FEV1 [49]. Although FD-based methods have the advantage of acquiring images during free tidal breathing, the present approach quantifies proton signal change during a vital capacity manoeuvre, thus enhancing the contrast among the low-ventilated regions $[32,50]$. Moreover, breath-hold acquisition minimises artefacts related to breathing irregularities and to through-plane motion during the acquisition [34, 48].

Third, our results show a moderate correlation between $\Delta^{1} \mathrm{H}-\mathrm{MRI}$ measures of ventilation and CF-specific structural score. With increasing morphological impairment, i.e. increasing CF score, there is a decrease in gas volume change and an increase in ventilation heterogeneity. Both CT and MRI have proven sensitivity to structural changes at various stages of CF lung disease [51, 52]. Nevertheless, previous studies have reported the presence of local functional abnormalities prior to the onset of structural injury [53]. Alterations in very small airways can result in uneven ventilation distribution, but such alterations may not be evident in MRI as structural impairments. Because the present study is cross-sectional, we can only speculate that MRI measures of ventilation will worsen with advancing structural alterations and that ventilation defects can anticipate morphological changes. Future longitudinal studies using multivolume MRI may elucidate local structure-function relationships in patients followed over several years.

The study has some limitations. First, the study design is cross-sectional and only longitudinal data points would determine the relationship between the progression of quantitative imaging biomarkers, lung disease progression and treatment regimen.

Second, the study includes a small number of patients and lacks healthy controls. Nevertheless, the range of disease is broad enough to capture data from subjects with minimal to advanced disease and patients with minimal disease have shown median ventilation values comparable to the ones previously reported in healthy controls [32]. 
Third, inspiratory and expiratory MRI is not spirometer guided. To introduce quantitative multivolume MRI into clinical practice, the implementation of lung volume guidance with a spirometer is an essential but feasible step to standardise imaging [54].

Another concern of the study is the potential for motion artefacts derived from the breath-hold requirement of 7-9 s. However, CF patients attend intensive respiratory physiotherapy programmes that allow them to perform the imaging examination without difficulty, as in our group.

In conclusion, we have found a strong correlation between quantitative multivolume MRI, spirometry and MBW and a clear structure-function relationship in CF lung disease. These results support the further investigation of multivolume MRI to detect and regionally monitor disease progression and to quantify individual response to treatment. As a non-ionising imaging technique, multivolume MRI represents a unique tool for longitudinal studies in CF care.

Conflict of interest: F. Pennati has nothing to disclose. C. Salito has nothing to disclose. I. Borzani has nothing to disclose. G. Cervellin has nothing to disclose. S. Gambazza reports fees for consulting on lung clearance index from Vertex Pharmaceuticals. R. Guarise has nothing to disclose. M.C. Russo has nothing to disclose. C. Colombo has nothing to disclose. A. Aliverti has nothing to disclose.

\section{References}

1 O'Sullivan BP, Freedman SD. Cystic fibrosis. Lancet 2009; 373: 1891-1904.

2 Gibson RL, Burns JL, Ramsey BW. Pathophysiology and management of pulmonary infections in cystic fibrosis. Am I Respir Crit Care Med 2003; 168: 918-951.

3 Wainwright CE. Ivacaftor for patients with cystic fibrosis. Expert Rev Respir Med 2014; 8: 533-538.

4 Ramsey BW, Banks-Schlegel S, Accurso FJ, et al. Future directions in early cystic fibrosis lung disease research: an NHLBI workshop report. Am J Respir Crit Care Med 2012; 185: 887-892.

5 Tiddens HA. Detecting early structural lung damage in cystic fibrosis. Pediatr Pulmonol 2002; 34: 228-231.

6 De Jong PA, Nakano Y, Lequin MH, et al. Progressive damage on high resolution computed tomography despite stable lung function in cystic fibrosis. Eur Respir J 2004; 23: 93-97.

7 Tiddens HA, Donaldson SH, Rosenfeld M, et al. Cystic fibrosis lung disease starts in the small airways: can we treat it more effectively? Pediatr Pulmonol 2010; 45: 107-117.

8 Bakker EM, Borsboom GJ, van der Wiel Kooij EC, et al. Small airway involvement in cystic fibrosis lung disease: routine spirometry as an early and sensitive marker. Pediatr Pulmonol 2013; 48: 1081-1088.

9 Bakker EM, Volpi S, Salonini E, et al. Improved treatment response to dornase alfa in cystic fibrosis patients using controlled inhalation. Eur Respir J 2011; 38: 1328-1335.

10 de Jong PA, Lindblad A, Rubin L, et al. Progression of lung disease on computed tomography and pulmonary function tests in children and adults with cystic fibrosis. Thorax 2006; 61: 80-85.

11 Lum S, Gustafsson P, Ljungberg H, et al. Early detection of cystic fibrosis lung disease: multiple-breath washout versus raised volume tests. Thorax 2007; 62: 341-347.

12 Aurora P, Bush A, Gustafsson P, et al. Multiple-breath washout as a marker of lung disease in preschool children with cystic fibrosis. Am J Respir Crit Care Med 2005; 171: 249-256.

13 Aurora P, Stanojevic S, Wade A, et al. Lung clearance index at 4 years predicts subsequent lung function in children with cystic fibrosis. Am J Respir Crit Care Med 2011; 183: 752-758.

14 Kraemer R, Blum A, Schibler A, et al. Ventilation in homogeneities in relation to standard lung function in patients with cystic fibrosis. Am J Respir Crit Care Med 2005; 171: 371-378.

15 Gustafsson PM, De Jong PA, Tiddens HA, et al. Multiple-breath inert gas washout and spirometry versus structural lung disease in cystic fibrosis. Thorax 2008; 63: 129-134.

16 Stahl M, Wielpütz MO, Graeber SY, et al. Comparison of lung clearance index and magnetic resonance imaging for assessment of lung disease in children with cystic fibrosis. Am J Respir Crit Care Med 2017; 195: 349-359.

17 Davies J, Sheridan H, Bell N, et al. Assessment of clinical response to ivacaftor with lung clearance index in cystic fibrosis patients with a G551D-CFTR mutation and preserved spirometry: a randomised controlled trial. Lancet Respir Med 2013; 1: 630-638.

18 Amin R, Subbarao P, Lou W, et al. The effect of dornase alfa on ventilation inhomogeneity in patients with cystic fibrosis. Eur Respir J 2011; 37: 806-812.

19 Ratjen F, Hug C, Marigowda G. Efficacy and safety of lumacaftor and ivacaftor in patients aged 6-11 years with cystic fibrosis homozygous for F508del-CFTR: a randomised, placebo-controlled, phase 3 trial. Lancet Respir Med 2017; 5: 557-567.

20 Brody AS, Klein JS, Molina PL, et al. High-resolution computed tomography in young patients with cystic fibrosis: distribution of abnormalities and correlation with pulmonary function tests. J Pediatr 2004; 145: 32-38.

21 Eichinger M, Heussel CP, Kauczor HU, et al. Computed tomography and magnetic resonance imaging in cystic fibrosis lung disease. J Magn Reson Imaging 2010; 32: 1370-1378.

22 O'Connell OJ, McWilliams S, McGarrigle A, et al. Radiologic imaging in cystic fibrosis: cumulative effective dose and changing trends over 2 decades. Chest 2012; 141: 1575-1583.

23 Stahl M, Wielpütz MO, Graeber SY, et al. Comparison of lung clearance index and magnetic resonance imaging for assessment of lung disease in children with cystic fibrosis. Am J Respir Crit Care Med 2017; 195: 349-359.

24 Wielpütz MO, Puderbach M, Kopp-Schneider A, et al. Magnetic resonance imaging detects changes in structure and perfusion, and response to therapy in early cystic fibrosis lung disease. Am J Respir Crit Care Med 2014; 189: 956-965.

25 De Jong PA, Ottink MD, Robben SG, et al. Pulmonary disease assessment in cystic fibrosis: comparison of CT scoring systems and value of bronchial and arterial dimension measurements. Radiology 2004; 231: 434-439.

26 Eichinger M, Puderbach M, Fink C, et al. Contrast-enhanced 3D MRI of lung perfusion in children with cystic fibrosis-initial results. Eur Radiol 2006; 16: 2147-2152. 

disease using MRI. Eur I Radiol 2012; 81: 1321-1329.

28 DeBoer EM, Swiercz W, Heltshe SL, et al. Automated CT scan scores of bronchiectasis and air trapping in cystic fibrosis. Chest 2014; 145: 593-603.

29 Goris ML, Zhu HJ, Blankenberg F, et al. An automated approach to quantitative air trapping measurements in mild cystic fibrosis. Chest 2003; 123: 1655-1663.

30 de Jong PA, Nakano Y, Hop WC, et al. Changes in airway dimensions on computed tomography scans of children with cystic fibrosis. Am J Respir Crit Care Med 2005; 172: 218-224.

31 Zapke M, Topf HG, Zenker M, et al. Magnetic resonance lung function - a breakthrough for lung imaging and functional assessment? A phantom study and clinical trial. Respir Res 2006; 7: 106.

32 Pennati F, Quirk J, Yablonskiy D, et al. Assessment of regional lung function by multi-volume $1 \mathrm{H}-\mathrm{MRI}$ in health and obstructive lung disease: comparison with 3He-MRI. Radiology 2014; 273: 580-590.

33 Bauman G, Puderbach M, Deimling M, et al. Non-contrast-enhanced perfusion and ventilation assessment of the human lung by means of Fourier decomposition in proton MRI. Magn Reson Med 2009; 62: 656-664.

34 Bauman G, Bieri O. Matrix pencil decomposition of time-resolved proton MRI for robust and improved assessment of pulmonary ventilation and perfusion. Magn Reson Med 2017; 77: 336-342.

35 Fischer A, Weick S, Ritter CO, et al. Self-gated non-contrast-enhanced functional lung imaging (SENCEFUL) using a quasi-random fast low-angle shot (FLASH) sequence and proton MRI. NMR Biomed 2014; 27: 907-917.

36 Quanjer PH, Stanojevic S, Cole TJ, et al. Multi-ethnic reference values for spirometry for the 3-95-yr age range: the global lung function 2012 equations. Eur Respir J 2012; 40: 1324-1343.

37 Miller MR, Hankinson J, Brusasco V, et al. Standardisation of spirometry. Eur Respir J 2005; 26: 319-338.

38 Jensen R, Green K, Gustafsson P, et al. Standard Operating Procedure: Multiple Breath Nitrogen Washout. Dürnten, Eco Medics AG, 2013

39 Robinson PD, Latzin P, Verbanck S, et al. Consensus statement for inert gas washout measurement using multiple and single breath tests. Eur Respir J 2013; 41: 507-522.

40 About MIPAV. Center for Information Technology. National Institutes of Health. 2007. http://mipav.cit.nih.gov/ Date last updated: April 5, 2013.

41 Lucas BD, Kanade T. An iterative image registration technique with an application to stereo vision. In: Proceedings of the International Joint Conference on Artificial Intelligence (IJCAI). Santa Clara, IJCAI Organization, 1981; pp. 674-679.

42 Pennati F, Roach DJ, Clancy JP, et al. Assessment of pulmonary structure-function relationships in young children and adolescents with cystic fibrosis by multivolume proton-MRI and CT. J Magn Reson Imaging 2018; 48: 531-542.

43 Verbanck S, Paiva M, Paeps E, et al. Lung clearance index in adult cystic fibrosis patients: the role of convection-dependent lung units. Eur Respir J 2013; 42: 380-388.

44 Aurora P, Gustafsson P, Bush A, et al. Multiple breath inert gas washout as a measure of ventilation distribution in children with cystic fibrosis. Thorax 2004; 59: 1068-1073.

45 Kirby M, Svenningsen S, Ahmed H, et al. Quantitative evaluation of hyperpolarized helium-3 magnetic resonance imaging of lung function variability in cystic fibrosis. Acad Radiol 2011; 18: 1006-1013.

46 Mentore K, Froh DK, de Lange EE, et al. Hyperpolarized He 3 MRI of the lung in cystic fibrosis: assessment at baseline and after bronchodilator and airway clearance treatment1. Acad Radiol 2005; 12: 1423-1429.

47 McMahon CJ, Dodd JD, Hill C, et al. Hyperpolarized 3helium magnetic resonance ventilation imaging of the lung in cystic fibrosis: comparison with high resolution CT and spirometry. Eur Radiol 2006; 16: 2483-2490.

48 Veldhoen S, Weng AM, Knapp J, et al. Self-gated non-contrast-enhanced functional lung MR imaging for quantitative ventilation assessment in patients with cystic fibrosis. Radiology 2017; 283: 242-251.

49 Nyilas S, Bauman G, Sommer G, et al. Novel magnetic resonance technique for functional imaging of cystic fibrosis lung disease. Eur Respir J 2017; 50: 1701464.

50 Bonnel AS, Song SMH, Kesavarju K, et al. Quantitative air-trapping analysis in children with mild cystic fibrosis lung disease. Pediatr Pulmonol 2004; 38: 396-405.

51 Ciet P, Serra G, Bertolo S, et al. Assessment of CF lung disease using motion corrected PROPELLER MRI a comparison with CT. Eur Radiol 2016; 26: 780-787.

52 Tepper LA, Ciet P, Caudri D, et al. Validating chest MRI to detect and monitor cystic fibrosis lung disease in a pediatric cohort. Pediatr Pulmonol 2016; 51: 34-41.

53 Donnelly LF, Gelfand MJ, Brody AS, et al. Comparison between morphologic changes seen on high-resolution CT and regional pulmonary perfusion seen on SPECT in patients with cystic fibrosis. Pediatr Radiol 1997; 27: 920-925.

54 Salamon E, Lever S, Kuo W, et al. Spirometer guided chest imaging in children: it is worth the effort! Pediatr Pulmonol 2017; 52: 48-56. 